University of Nebraska - Lincoln

DigitalCommons@University of Nebraska - Lincoln

Entomology Papers from Other Sources

Entomology Collections, Miscellaneous

1998

An Immunomarking Technique for Labeling Minute Parasitoids

James Hagler

USDA-ARS

Charles Jackson

USDA-ARS

Follow this and additional works at: https://digitalcommons.unl.edu/entomologyother

Part of the Entomology Commons

Hagler, James and Jackson, Charles, "An Immunomarking Technique for Labeling Minute Parasitoids" (1998). Entomology Papers from Other Sources. 66.

https://digitalcommons.unl.edu/entomologyother/66

This Article is brought to you for free and open access by the Entomology Collections, Miscellaneous at DigitalCommons@University of Nebraska - Lincoln. It has been accepted for inclusion in Entomology Papers from Other Sources by an authorized administrator of DigitalCommons@University of Nebraska - Lincoln. 
Biological CONTROL

\title{
An Immunomarking Technique for Labeling Minute Parasitoids
}

\author{
JAMES R. HAGLER AND CHARLES G. JACKSON
}

Western Cotton Research Laboratory, USDA-ARS, 4135 East Broadway Road, Phoenix, AZ 85040

\begin{abstract}
Environ. Entomol 27(4): 1010-1016 (1998)
ABSTRACT A laboratory study was conducted to examine the efficacy of a novel immunomarking technique on Anaphes iole Girault, a minute parasitoid of Lygus spp. eggs. Adult A. iole were marked with the readily available mammal protein, rabbit immunoglobulin $\mathrm{G}$ (IgG), by 3 different application methods. Adult parasitoids were marked internally by feeding them a honey solution spiked with rabbit IgG and externally by contact exposure or topical mist. Marked individuals were then assayed using a sandwich enzyme-linked immunosorbent assay (ELISA) for the presence of the IgG marker using an antibody specific to rabbit IgG (antirabbit IgG developed in goat). Data indicate that the IgG marker was retained throughout the entire adult lifespan in almost (98.9\%) every individual parasitoid assayed, regardless of the application method used. The advantages and limitations of using immunomarkers for mark-release-recapture studies involving minute insects are discussed.
\end{abstract}

KEY WORDS Anaphes iole, Lygus spp., mark-release-recapture, dispersal, parasitoid, enzymelinked immunosorbent assay

DiSPERSAL BEHAVIOR SUCH as immigration and inter- and intra-field crop movement must be precisely monitored to understand the population dynamics of insect parasitoids. Generally, parasitoid dispersal has been researched using mark-release-recapture techniques that involve marking insects with an environmentally persistent material, releasing them into the field, and recapturing them after a given time interval. A wide variety of materials have been used to mark insects for dispersal studies (Southwood 1978, Akey et al. 1991). However, marking parasitoids has proven to be a special challenge for biological control researchers because most parasitoids are very small and delicate. Conventional marking materials such as paints, dyes, dusts, and tags are ineffective on small parasitoids because they are heavy and can inhibit normal dispersal behavior (Steiner 1965).

Probably the best markers to date for studying parasitoid dispersal have been the trace elements (Jackson et al. 1988, Jackson and Debolt 1990, Akey et al. 1991). These are environmentally safe and relatively persistent in insect tissues. Studies have shown that parasitoids can be effectively marked with rubidium $(\mathrm{Rb})$. For example, Anaphes ovijentatus (Crosby \& Leonard) (=A. iole Girault), a Lygus spp. egg parasitoid, was successfully marked with $\mathrm{Rb}$ when reared from labeled eggs of lygus adults fed an artificial diet containing $>500$ ppm RbCl (Jackson et al. 1988). In a similar experiment, Microplitis croceipes (Cresson) was marked by rearing it in host larvae that fed on an artificial diet containing 4 different trace elements (Hopper and Woolson 1991). However, there are sev-

This article reports the results of research only. Mention of a proprietary product does not constitute an endorsement or a recommendation by USDA for its use. eral drawbacks to using trace elements as biological markers. Rubidium retention in some insects declines rapidly after removal of the insect from the $\mathrm{Rb}$-enriched host (Fleischer et al. 1986, Hopper and Woolson 1991) or after it feeds on a potassium-rich food (Fairbanks and Burch 1968). Some studies have reported that high levels of Rb can adversely affect the development of certain insects (Stimmann et al. 1973, Van Steenwyk et al. 1978). For example, Jackson et al. (1988) determined that A. iole that developed in eggs of Lygus hesperus Knight reared on diet containing high concentrations of $\mathrm{RbCl}$ (1,000 ppm) had a shorter lifespan and lower fecundity than those reared on lower concentrations of $\mathrm{RbCl}$. Besides the negative effects of $\mathrm{Rb}$ on parasitoid biology, the detection of elemental labels is difficult. Trace element analyses require technical expertise and an expensive atomic absorption spectrophotometer. Furthermore, the analyses of samples for the presence of the trace element is extensive, time consuming, and generates hazardous waste (Akey and Burns 1991).

Recently, a simple, rapid, safe, inexpensive, and stable marking procedure was developed to mark phytophagous and predaceous insects. We externally marked adult $L$. hesperus and convergent lady beetles, Hippodamia convergens Guérin-Méneville, with a rabbit immunoglobulin $\mathrm{G}$ (IgG) solution. In the laboratory, rabbit IgG, a mammalian protein, remained detectable on $L$. hesperus for over a week after marking (Hagler et al. 1992a). In the field, rabbit IgG remained detectable on $H$. convergens for at least $4 \mathrm{wk}$ under extreme temperatures $\left(>40^{\circ} \mathrm{C}\right)$ and rainfall $(>2.54$ $\mathrm{cm})$ (Hagler 1997). Because the IgG marking procedure worked so well on these insects, we planned to investigate the feasibility of immunomarking very small parasitoids. 
This study was designed to test the feasibility of labeling the minute Lygus spp. egg parasitoid, A. iole, with rabbit IgG. We examined the efficacy and retention of rabbit IgG using 3 different marking procedures. First, we marked adults internally by feeding them a honey solution spiked with rabbit IgG. Second, we marked adult $A$. iole by exposing them to a wetted filter paper containing rabbit IgG. Finally, we marked adults by spraying them with a topical mist spray. A further study was conducted to determine if proteinmarked individuals can transfer the protein to unmarked individuals.

\section{Materials and Methods}

Test Insects. Anaphes iole were received from a commercial insectary (BioTactics, Riverside, CA) as pupae in L. hesperus eggs. The eggs had been removed from oviposition packets (Patana 1982) and placed onto organdy cloth held between layers of moistened paper towels to prevent the eggs from desiccating during shipment. We put the parasitized eggs into a black emergence box similar to the one described by Graham et al. (1986). Emerging parasitoids were collected in clear, lighted snapvials that were attached to the side of the box.

Parasitoid Marking Procedures. Three different methods were used to label the parasitoids: (1) ingestion (internal label), (2) contact exposure (external label) and, (3) topical contact mist (external label). Newly emerged adult parasitoids were held in cages for $1-2 \mathrm{~h}$ before marking. Cages were made from $33-\mathrm{ml}$ snapvials with the bottom removed and screened with organdy cloth.

Ingestion. Newly emerged adult parasitoids were held without food or water for 1-2 h, then were fed a spiked honey solution containing $2.5 \mathrm{mg}$ rabbit IgG per milliliter of honey (Sigma, St. Louis, MO, No. I5006). The spiked honey was streaked across the organdy screened opening of the vials, and the parasitoids were allowed to feed for $1 \mathrm{~h}$ before being transferred to a clean cage containing regular (unlabeled) honey. The marked parasitoids were held in an environmental chamber set at $28 \pm 1.1^{\circ} \mathrm{C}, 60 \% \mathrm{RH}$, and a photoperiod of 14:10 (L:D) h after feeding on the spiked honey solution. Approximately 30 live parasitoids were removed daily throughout their adult lifespan $(\approx 8 \mathrm{~d})$ from the environmental chamber and frozen $\left(-70^{\circ} \mathrm{C}\right)$. The frozen parasitoids were individually homogenized in $150 \mu \mathrm{l}$ phosphate buffered saline (PBS) and assayed for the presence of the rabbit IgG immunomarker by the sandwich ELISA described below.

Contact. The parasitoids were allowed to feed on a regular honey solution for 1-2 $\mathrm{h}$ to reduce the amount of feeding on the labeled solution. A Whatman No.1 qualitative filter paper $(5.5 \mathrm{~cm})$ was placed in the lid of a plastic petri dish ( 60 by $15 \mathrm{~mm})$. The filter paper was soaked with $450 \mu \mathrm{l}$ of a $5.0 \mathrm{mg} / \mathrm{ml} \mathrm{rabbit} \mathrm{IgG}$ solution delivered by micropipette. The parasitoids were immobilized in a freezer for 1-2 min, then tapped onto the wetted filter paper and the petri dish was covered. After a 1-h exposure (walking) on the wetted filter paper, the parasitoids were transferred into a clean cage containing regular honey and placed into the environmental chamber described above. Approximately 30 live parasitoids were removed daily (for $8 \mathrm{~d}$ ) from the environmental chamber and frozen for later analyses. The frozen parasitoids were then homogenized in $150 \mu \mathrm{l}$ PBS and assayed for the presence of the rabbit IgG immunomarker by the sandwich ELISA described below.

Topical Contact by Misting. Newly emerged parasitoids were held in cages for $1-2 \mathrm{~h}$ with regular honey, then transferred to a larger screened vial $(180 \mathrm{ml}, 49$ by $110 \mathrm{~mm}$ ). They were misted with $5.0 \mathrm{mg} / \mathrm{ml} \mathrm{rabbit}$ IgG solution with a perfume atomizer until there was a fine mist on the screen and inside the vial. The vial was then held in an airstream until the mist had dried $(\approx 3-5 \mathrm{~min})$. After drying, the parasitoids were transferred to a clean cage containing regular honey and placed into the environmental chamber described above. Approximately 30 live parasitoids were removed daily (for $8 \mathrm{~d}$ ) from the environmental chamber and frozen for later analyses. The frozen parasitoids were then homogenized in $150 \mu \mathrm{l}$ PBS and assayed for the presence of the rabbit IgG immunomarker by the sandwich ELISA described below.

Cross Contamination Test. A study was conducted to determine if the protein marker transferred from marked individuals to unmarked ones. Adult males were marked by the contact marking method described above. The males were then placed into a clean cage containing regular honey and placed in the environmental chamber described above. After $24 \mathrm{~h}$, the marked males were transferred to a 2 nd clean vial and unmarked females were put into the vial with them. These parasitoids were removed from the cage after $24 \mathrm{~h}$ and frozen for later analyses. The frozen individuals were sexed, homogenized in $150 \mu \mathrm{l}$ PBS and assayed for the presence of the rabbit IgG protein by the sandwich ELISA described below.

ELISA Procedure. A sandwich ELISA was performed on each adult $A$. iole as described by Hagler et al. (1992a). Each well of a 96-well ELISA microplate was coated with $100 \mu \mathrm{l}$ of antirabbit IgG (developed in goat) (Sigma, No. R2004) diluted 1:500 in doubledistilled $\mathrm{H}_{2} \mathrm{O}$ and incubated overnight at $4^{\circ} \mathrm{C}$ (note: this incubation time can be as short as $1 \mathrm{~h}$ without loss of efficacy). The antirabbit IgG was discarded and 360 $\mu$ l of $1 \%$ nonfat dry milk in double-distilled $\mathrm{H}_{2} \mathrm{O}$ was added to each well for $30 \mathrm{~min}$ at $27^{\circ} \mathrm{C}$ to block any remaining nonspecific binding sites on the plates. After the nonfat milk was removed, a $150-\mu \mathrm{l}$ aliquot of the homogenized parasitoid sample was placed in each well of the pretreated assay plate and incubated overnight at $4^{\circ} \mathrm{C}$ (note: in this reaction too, the incubation time can be as short as $1 \mathrm{~h}$ without loss of efficacy). Parasitoid samples were then discarded and each well was briefly rinsed 3 times with PBS Tween 20 (0.05\%) and twice with PBS. Aliquots $(50 \mu \mathrm{l})$ of antirabbit IgG conjugated to horseradish peroxidase (Sigma, No. A-6154) diluted to $1: 1,000$ in $1.0 \%$ nonfat milk, were added to each well for $1 \mathrm{~h}$ at $27^{\circ} \mathrm{C}$. Plates were again washed as described above, and $50 \mu$ l of substrate was 


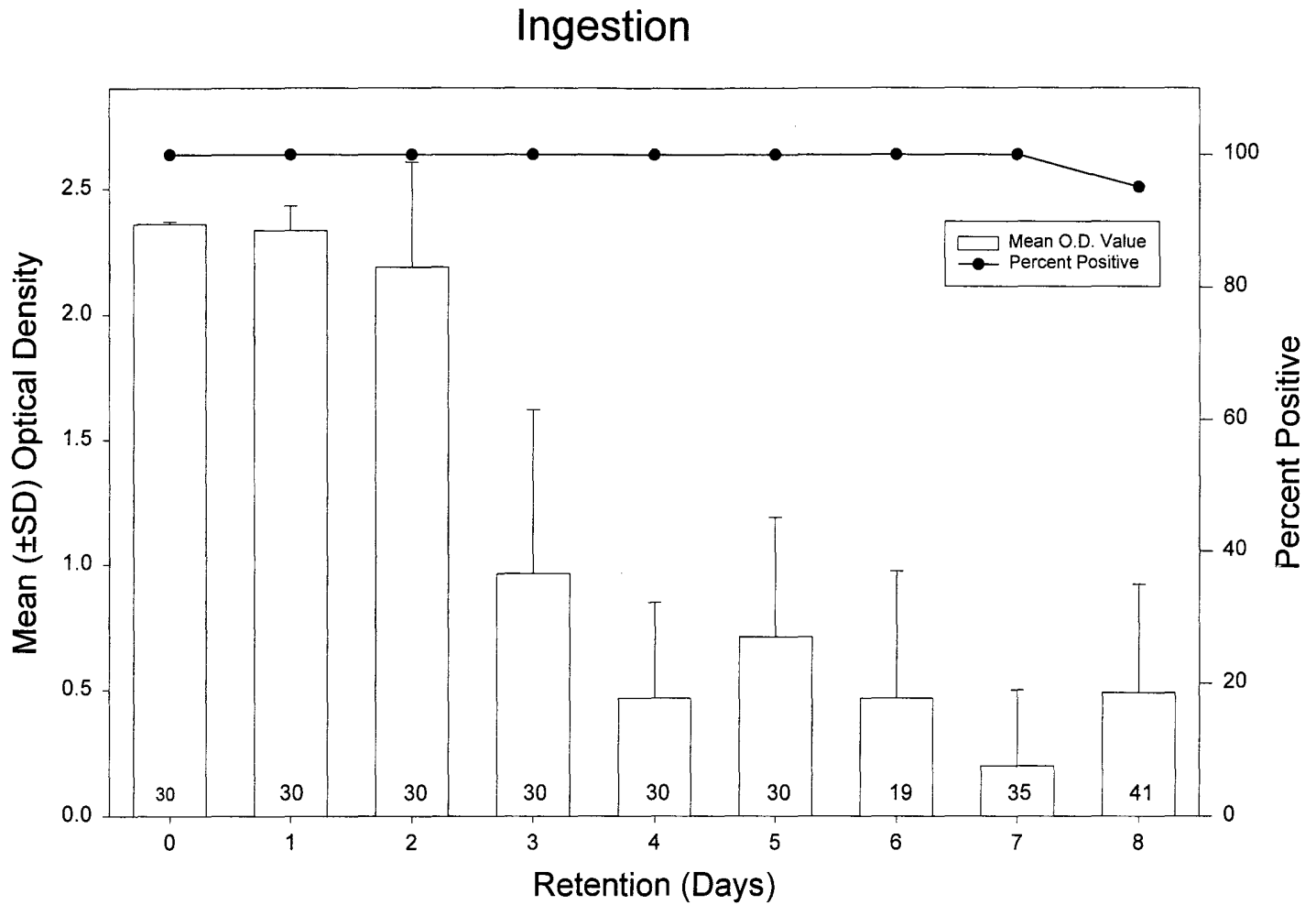

Fig. 1. The mean \pm SD ELISA optical density values (left $y$-axis) and percentage of adult $A$. iole scoring positive (right $y$-axis) for the presence of rabbit IgG immunolabel after feeding on honey containing rabbit IgG. The number inside each vertical bar is the sample size for that treatment.

added using the reagents supplied in a horseradish peroxidase substrate kit (Bio-Rad, Richmond, CA, No. 172-1064). Following substrate incubation (2 h), the optical density of each sample was measured with a microplate reader set at $405 \mathrm{~nm}$. The mean $( \pm \mathrm{SD}$ ) ELISA optical density value and the percentage of parasitoids scoring positive for IgG remains was tallied for each date.

Negative Parasitoid Controls. Adult A. iole known not to contain any immunomarker were assayed by the sandwich ELISA described above $(n=262)$. Parasitoids known to have been exposed to rabbit IgG were scored positive for the presence of the immunomarker if the optical density value exceeded the mean negative control reading by 3 standard deviations (critical value) (Schoof et al. 1986, Hagler et al. 1992b).

\section{Results}

Retention of IgG by Ingestion. The mean $( \pm S D)$ ELISA optical density values and percentage of parasitoids scoring positive for rabbit IgG remains after ingesting a honey solution containing rabbit IgG are given in Fig. 1. Mean ELISA optical density values ranged from 2.38 on day 0 to 0.20 on day 7 . Virtually all of the individuals (99.3\%) that fed on the spiked IgG honey solution yielded a positive immunoreaction (with exception of 2 individuals on day 8 ) throughout their adult lifespan. There was a considerable decrease in the amount of rabbit IgG detected in the parasitoids after $2 \mathrm{~d}$; however, most of the individuals still contained easily identifiable amounts of rabbit IgG. For example, on day 7 an average immunoreaction yielded an ELISA optical density value of 0.20 . This value is $>100$ times greater than the mean negative control value $(-0.002 \pm 0.01)$ and almost 10 times greater than the conservative (mean $+3 \mathrm{SD}$ ) critical value $(0.028)$ used to score positive immunoreactions.

Retention of IgG by Contact Exposure. The mean $( \pm$ SD) ELISA optical density values and percentage of individuals scoring positive for rabbit IgG after contact exposure to the wetted filter paper are given in Fig. 2. Mean ELISA optical density values ranged from 2.32 on day 0 to 0.18 on day 2 . All of the individuals that walked on the IgG-soaked filter paper (with the exception of 1 individual on day 2) scored positive for IgG remains. Again, there was considerable day-to-day variation in the amount of IgG retained on the parasitiods, particularly beyond the 1-d holding interval. However, $99.6 \%$ of all individuals assayed by ELISA contained easily recognizable amounts of rabbit IgG on their body.

Retention of IgG by Topical Mist. The mean $( \pm S D)$ ELISA optical density values and percentage of individuals scoring positive for rabbit IgG after receiving a topical mist spray of rabbit IgG are given in Fig. 3. 


\section{Contact Exposure}

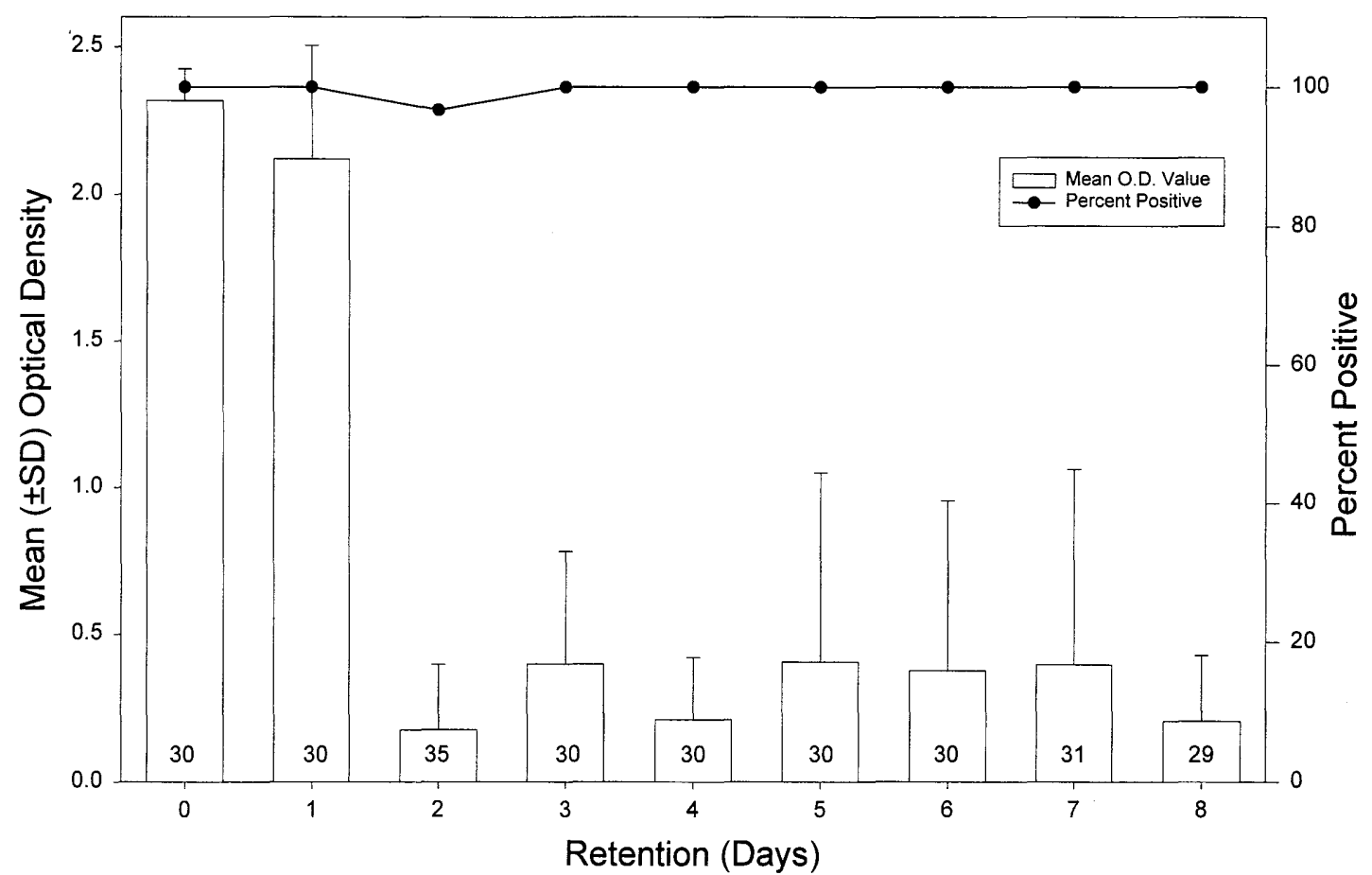

Fig. 2. The mean \pm SD ELISA optical density values (left y-axis) and percentage of adult $A$. iole scoring positive (right $y$-axis) for the presence of rabbit IgG immunolabel after contact exposure to rabbit IgG solution. The number inside each vertical bar is the sample size for each treatment.

Mean ELISA optical density values ranged from 2.24 on day 0 to 0.19 on day 6 . As with the previous marking methods, there was considerable day-to-day variation in the amount of IgG retained by the parasitoids. However, most $(97.6 \%)$ of the parasitoids that were sprayed with the IgG solution yielded positive immunoreactions for the presence of rabbit IgG.

Cross Contamination Test. The mean ( \pm SD) ELISA optical density values and percentage of males (marked) and females (unmarked) scoring positive for rabbit IgG are given in Table 1. The marked males and unmarked females yielded mean ELISA readings of 0.30 and -0.01 , respectively. Most of the adult males yielded a positive ELISA response (90\%) for the presence of the protein marker whereas only 1 of the females $(10 \%)$ was positive (Table 1).

\section{Discussion}

Immunomarkers have been used before to label large phytophagous and predaceous insect species (Hagler et al. 1992a, Hagler 1997). Immunomarkers also have been used to label prey for predation studies (Hagler and Durand 1994). In this study we report for the first time that immunomarkers can be used to successfully mark minute parasitoids for markrelease-recapture studies. This immunomarking procedure should expedite future field studies aimed at determining parasitoid dispersal patterns. Immunomarking parasitoids offers many practical advantages over many of the more conventional marking techniques (Southwood 1978). Colored paints, dyes, and dusts are usually too big or heavy for minute parasitoids. To date, trace elements have been the most effective markers for small parasitoid species (Akey et al. 1991). However, the immunomarking technique described here offers many advantages over trace element marking. For instance, thousands of parasitoids can be quickly labeled with small quantities of the inexpensive immunolabel. The ELISA procedure is also simple, rapid, and economical. A single person with only minimal training $(2 \mathrm{~d})$ can assay $>1,000$ individual parasitoids per day. Moreover, laboratory equipment needed to run an ELISA can be found in most laboratories, and ELISA reagents are inexpensive and safe. Although an ELISA microplate reader was used to quantify ELISA optical density readings, the qualitative nature of the assay combined with the clarity of the negative control parasitoids (i.e., no background coloration) does not warrant the purchase of one.

All 3 immunomarking techniques examined in this study proved to be equally effective for labeling adult parasitoids. Overall, only 10 of the 904 parasitoids marked yielded false negative immunoreactions. This suggests that $98.9 \%$ of the individuals marked with 


\section{Misted}

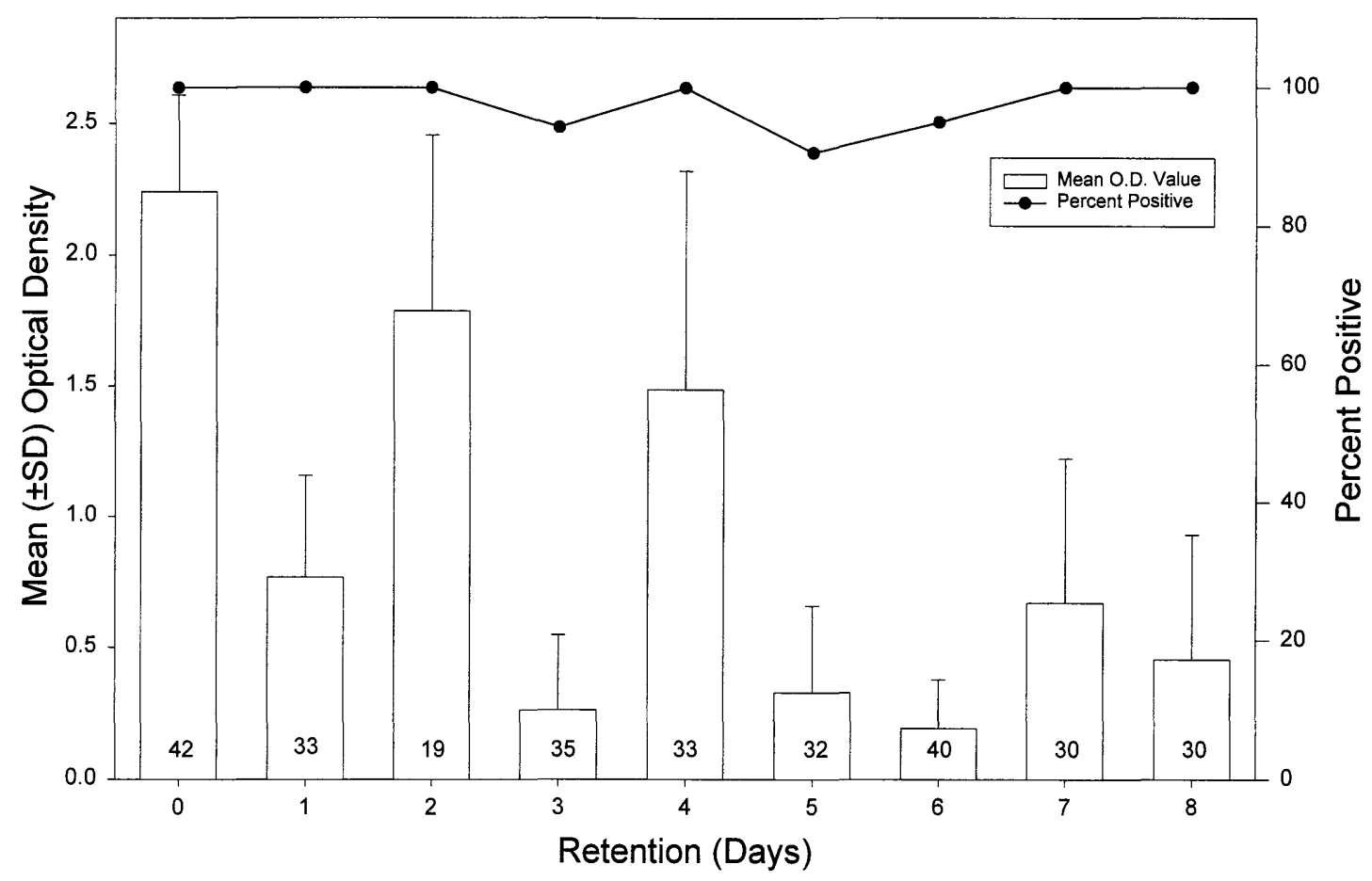

Fig. 3. The mean \pm SD ELISA optical density values (left $y$-axis) and percentage of adult A. iole scoring positive (right $y$-axis) for the presence of rabbit IgG immunolabel after exposure to a topical mist application of rabbit IgG solution. The number inside each vertical bar is the sample size for each treatment.

rabbit IgG, regardless of the time elapsed after marking (for up to $8 \mathrm{~d}$ ), yielded positive immunoreactions. Conversely, the negative control parasitoids yielded ELISA optical density values below zero $(-0.002 \pm$ 0.01 ). The low optical density values yielded by the unmarked parasitoids suggests that there is no cross reactivity between the antirabbit IgG and any of the proteins present in the adult parasitoids.

Anaphes iole proved to be an ideal model insect for this study because emerging adults readily feed on honey solution. Therefore, we were confident that most (all) of the adult parasitoids fed on the spiked honey solution shortly after emergence. There are several advantages of labeling insects by internal ingestion of foodstuffs. First, no handling of the insects is needed. This is extremely important when handling small, delicate parasitoids. Second, only a minute amount of the immunomarker is needed. We used $<500 \mu \mathrm{l}$ of honey (at $2.5 \mathrm{mg}$ rabbit IgG/ $\mathrm{ml}$ honey) for this experiment. Third, internal markers have less of a chance of effecting the normal behavior of the parasitoids. Extra care was needed for marking parasitoids by contact or topical mist. Finally, internal markers will not degrade under extreme field conditions. Internal markers are less vulnerable to washing off during rainstorms or degrading under intensive heat or humidity (see below for further discussion).

The fact that the internal marker remained detectable in the parasitoids throughout their adult lifespan $(\approx 8 \mathrm{~d})$ was surprising. It appears that rabbit IgG is slowly digested by adult parasitoids. While the amount of rabbit IgG detected generally declined overtime, the optical density values yielded $8 \mathrm{~d}$ after feeding were still $>250$ times greater than that of the mean negative control value (Fig. 1). In a previous study, we fed Geocoris punctipes (Say) an artificial diet spiked with rabbit IgG. These predators retained the IgG in their gut for $2.5 \mathrm{~d}$ when assayed by a rather insensitive

Table 1. ELISA results testing for the presence of rabbit IgG protein on unmarked females after exposure to marked males for 24 h

\begin{tabular}{|c|c|c|c|c|c|}
\hline Protein-marked males & 10 & $0.300(0.315)$ & $0.003-1.029$ & 0.011 & 90 \\
\hline
\end{tabular}

${ }^{a}$ Negative control mean ELISA reading $+3 \mathrm{SD}$. 
single radial immunodiffusion assay (Hagler and Cohen 1990). Apparently, the digestive enzymes present in both $G$. punctipes and A. iole do not destroy the integrity of the rabbit IgG after ingestion.

Further results from this study suggest that $A$. iole also can be successfully marked externally by contact exposure. Parasitoids that were marked by contact exposure on wetted filter paper probably picked up most of the marker by tarsal or antennal contact. However, some of the individuals also were observed drinking the marking solution. While the above-mentioned internal marking procedure worked to our satisfaction on the 1st attempt, several optimization studies were needed for the contact marking method. In early pilot tests we tended to oversaturate the filter paper (i.e., $>500 \mu \mathrm{l}$ ) with the rabbit IgG solution. This resulted in high parasitoid mortality from drowning. However, once the optimal volume of marking solution was determined $(450 \mu \mathrm{l})$, the contact application method was very effective (i.e., high proportion of positive responses with minimal mortality).

The other method we examined for externally marking parasitoids was by topical spray (mist). Parasitoids marked with the topical mist probably picked up most of the marker on their dorsal surfaces. However, exposure by tarsal or antennal contact or by drinking the misted solution probably occurred even though we tried to minimize surface contact and ingestion by rapidly drying the vials in an air stream. This method also required some optimization tests before we achieved the desired results. Several spray apparatuses were tried before we used the perfume spray bottle. The perfume atomizer yielded a fine, evenly distributed spray mist. Most of the misters we tried initially yielded spray droplets that were unevenly distributed and too large. In such cases, many of the parasitoids were not exposed to the marking solution and others were damaged or killed by excessive wetting. Another misting apparatus that showed considerable promise for topically marking $A$. iole was a medical nebulizer, such as those commonly used by asthmatic patients. In a pilot test, we nebulized A. iole and held them for only $2 \mathrm{~d}$ after marking. All $(n=66)$ of those parasitoids marked with the nebulizer showed positive for the presence of rabbit IgG when assayed by ELISA (data not shown). While the perfume bottle atomizer was inexpensive and effective for marking $A$. iole, a nebulizer might be advantageous in some situations. For example, nebulizers produce an extremely fine, foglike mist that requires even less rabbit IgG solution than a more conventional mist sprayer. Furthermore, the fine mist spray decreases the chance of the marker affecting the behavior or longevity of the parasitoids and provides a more uniform distribution of the marker on the delicate parasitoids. The major disadvantage of using a nebulizer is that it is more expensive (approximately $\$ 120.00$ ) than a cheap perfume sprayer.

The cross-contamination test showed that there is a possibility that protein-marked parasitoids can transfer the protein to unmarked parasitoids. One of the $\mathbf{1 0}$ unmarked females scored positive for the presence of rabbit protein. However, the ELISA reading yielded by this individual was only slightly above that of the negative control threshold (Table 1). The source of the protein transferred to this single individual is unknown. Perhaps she obtained the protein by direct contact with marked males (e.g., mating or trophallaxis), by direct contact with a vial that might have been contaminated by marked males, or by a combination of these factors. All of these potential sources for contamination are areas that deserve further investigation.

Potential limitations to immunomarking insects help identify areas for further investigation. Parasitoids may lose the IgG marker while maturing from one life stage to another. We avoided this potential problem by studying adult parasitoids. Another potential limitation is that parasitoids may lose the external marker under extreme field conditions. For example, the rabbit IgG marking protein might wash off during rainstorms or denature under high temperatures. The chances of this happening are minimized by marking the parasitoids internally by feeding them a honey solution containing rabbit IgG. Parasitoids marked by external contact or topical mist could lose their marker as a result of extreme weather conditions. However, a previous study showed that IgG proteins (rabbit IgG and chicken IgG) that were topically sprayed on $H$. convergens remained on them for $>3 \mathrm{wk}$ under extreme field conditions (Hagler 1997). This long retention time (i.e. $>25 \mathrm{~d}$ ) associated with immunolabels is superior to that reported for elemental markers and dusts (Van Steenwyk 1991).

Another advantageous characteristic of using immunolabels for mark-release-recapture studies is their specificity. Multiple immunomarkers can be used to mark different cohorts of individuals using different vertebrate-specific proteins. For example, in another study we determined that rabbit IgG and chicken IgG antibodies do not cross-react in a sandwich ELISA Therefore different IgGs can be used to mark different cohorts of individuals (unpublished data).

An important area for future research includes examination of the movement of immunolabels through the food web. It has been demonstrated that rabbit IgG can be used to label insect prey for predation studies (Hagler and Durand 1994). However, it has not been demonstrated that host plants can be effectively marked with immunolabels. Levi (1970) reported that plants treated with $\mathrm{Rb}$ immediately absorb and translocate $\mathrm{Rb}$ throughout their system. Subsequently, adult lepidopteran pests that fed on Rb-treated host plants during their larval stage retained $\mathrm{Rb}$ as they matured (Berry et al. 1972, Stimmann 1974).

The potential sublethal effects of this mark-releaserecapture technique deserve further investigation. Although we did not investigate the sublethal effects of the immunomarker in this study, it did not appear that the parasitoids were affected by the IgG marker (once we optimized the marking technique). However, studies are needed to determine if vertebrate IgGs affect parasitoid longevity, fecundity, mobility, and so on. We are initiating such studies in our laboratory. 
In summary, the findings from this study suggest that rabbit IgG can be used to internally or externally mark adult $A$. iole. This immunomarking procedure and its accompanying ELISA are inexpensive (require very little specialized equipment), are environmentally safe (do not generate hazardous waste), and are technically easy (require minimal personnel training and sample preparation). The best method for applying immunomarkers will ultimately depend on the nature of the experiment, the characteristics of the parasitoid being marked, and the preference of the investigator. For A. iole, the best method was marking them internally by feeding them a honey solution containing rabbit IgG. However, for parasitoid species that do not readily feed on honey (or any other solution), the contact exposure or topical mist methods should work satisfactorily in most situations. Additionally, for extremely delicate parasitoid species (i.e., Trichogramma spp.) we recommend marking them topically with a medical nebulizer or Potter spray tower.

We expect to use this assay for further parasitoid dispersal studies. The immunoassay's specificity, sensitivity, and persistence suggests that we can label in vitro-reared parasitoids, release them in the field, and recapture them to examine their dispersal behavior. Moreover, by using multiple vertebrate-specific immunolabels, the intercrop movements of parasitoids can be examined easily. This novel immunolabeling procedure will expedite research aimed at evaluating insect pest and natural enemy dispersal patterns.

\section{Acknowledgments}

We thank Angela Chan, Sunny Carrington, Melani Charney, Patrick Dockens, Dan Langhorst, Scott Machtley, Lillian Moug, and Theresa Rodriguez for their meticulous technical assistance. Special thanks go to Debbie Hagler, Molly Hunter,

Marshall Johnson, Michael Smith, Sujaya Udayagiri, and Steve Welter for helpful reviews on early versions of the manuscript.

\section{References Cited}

Akey, D. H., and D. W. Burns. 1991. Analytical considerations and methodologies for elemental determinations in biological samples, pp. 25-36. In D. H. Akey, J. L. Hayes, and S. J. Fleischer [eds.], Use of elemental markers in the study of arthropod movement and trophic interactions. Southwestern Entomological Society, Dallas, TX.

Akey, D. H., J. L. Hayes, and S. J. Fleischer. 1991. Use of elemental markers in the study of arthropod movement and trophic interactions. Southwestern Entomological Society, Dallas, TX.

Berry, W. L., M. W. Stimmann, and W. W. Wolf. 1972. Marking of native phytophagous insects with rubidium: a proposed technique. Ann. Entomol. Soc. Am. 65: 236-238.

Fairbanks, L. D., and G. E. Burch. 1968. Whole body turnover of potassium traced by radiorubidium in adult Drosophila (Drosophilidae) and Megaselia (Phoridae). J. Insect Physiol. 14: 819-830.

Fleisher, S. J., M. J. Gaylor, N. V. Hue, and L. C. Graham. 1986. Uptake and elimination of rubidium, a physiolog- ical marker in adult Lygus lineolaris (Hemiptera: Miridae). Ann. Entomol. Soc. Am. 79: 19-25.

Graham, H. M., C. G. Jackson, and J. W. Debolt. 1986. Lygus spp. (Hemiptera: Miridae) and their parasites in agricultural areas of southern Arizona. Environ. Entomol. 15: 132-142.

Hagler, J. R. 1997. Evaluation of the field retention of a novel mark-release-recapture method. Environ. Entomol. 26: 1079-1086.

Hagler, J. R., and A. C. Cohen. 1990. Effects of time and temperature on digestion of purified antigen by Geocoris punctipes (Hemiptera: Lygaeidae) reared on artificial diet. Ann. Entomol. Soc. Am. 83: 1177-1180.

Hagler, J. R., and C. M. Durand. 1994. A new method for immunologically marking prey and its use in predation studies. Entomophaga. 39: 257-265.

Hagler, J. R., A. C. Cohen, D. Bradley-Dunlop, and F. J. Enriquez. 1992a. New approach to mark insects for feeding and dispersal studies. Environ. Entomol. 21: 20-25.

1992b. Field evaluation of predation on Lygus hesperus using a species- and stage-specific monoclonal antibody. Environ. Entomol. 21: 896-900.

Hopper, K. R., and E. A. Woolson. 1991. Labeling a parasitic wasp, Microplitis croceipes (Hymenoptera: Braconidae), with trace elements for mark-recapture studies. Ann. Entomol. Soc. Am. 84: 255-262.

Jackson, G. G., A. C. Cohen, and C. L. Verdugo. 1988. Labeling Anaphes ovijentatus (Hymenoptera: Mymaridae), an egg parasite of Lygus spp. (Hemiptera: Miridae) with rubidium. Ann. Entomol. Soc. Am. 81: 919-922.

Jackson, C. G., and J. W. Debolt. 1990. Labeling of Leiophron uniformis, a parasite of Lygus spp., with rubidium. Southwest. Entomol. 15: 239-243.

Levi, E. 1970. Penetration, retention, and transport of foliarapplied simple salts of $\mathrm{Na}, \mathrm{K}, \mathrm{Rb}$, and Cs. Physiol. Plant. 23: $811-819$.

Patana, R. 1982. Disposable diet packet for feeding and oviposition of Lygus hesperus (Hemiptera: Miridae). J. Econ. Entomol. 75: 668-669.

Schoof, D. D., S. Palchick, and C. H. Tempelis. 1986. Evaluation of predator-prey relationships using an enzyme immunoassay. Ann. Entomol. Soc. Am. 79: 91-95.

Southwood, T.R.E. 1978. Ecological methods, 2nd ed. Chapman \& Hall, London.

Steiner, L. F. 1965. A rapid method for identifying dyemarked fruit flies. J. Econ. Entomol. 58: 374-375.

Stimmann, M. W. 1974. Marking insects with rubidium: imported cabbageworm marked in the field. Environ. Entomol. 3: 327-328.

Stimmann, M. W., W. W. Wolf, and W. L. Berry. 1973 Cabbage loopers: biological effects of rubidium in the larval diet. J. Econ. Entomol. 66: 324-326.

Van Steenwyk, R. A. 1991. The uses of elemental marking for insect dispersal and mating competitiveness studies: from the laboratory to the field, pp. 15-23. In D. H. Akey, J. L. Hayes, and S. J. Fleischer [eds.], Use of elemental markers in the study of arthropod movement and trophic interactions. Southwestern Entomological Society, Dallas, TX

Van Steenwyk, R. A., G. R. Ballmer, A. L. Page, and H. T. Reynolds. 1978. Marking pink bollworm with rubidium. Ann. Entomol. Soc. Am. 71: 81-84.

Received for publication 18 July 1997; accepted 18 March 1998 